\title{
Colitis induced in mice with dextran sulfate sodium (DSS) is mediated by the NLRP3 inflammasome
}

\author{
Christian Bauer, ${ }^{1}$ Peter Duewell, ${ }^{1}$ Christine Mayer, ${ }^{1}$ Hans Anton Lehr, ${ }^{2}$ \\ Katherine A Fitzgerald, ${ }^{3}$ Marc Dauer, ${ }^{4}$ Jurg Tschopp, ${ }^{5}$ Stefan Endres, ${ }^{6}$ \\ Eicke Latz, ${ }^{3}$ Max Schnurr ${ }^{1}$
}

\begin{abstract}
- Additional figures are published online only. To view these files please visit the journal online (http://gut.bmj. com)

${ }^{1}$ Department of Internal Medicine, University of Munich, Munich, Germany Institut Universitaire de Pathologie, Universite de Lausanne, Lausanne,

Switzerland

${ }^{3}$ Department of Infectious Diseases and Immunology, University of Massachusetts Medical School, Worcester, Massachusetts, USA ${ }^{4}$ Department of Medicine II, Saarland University Hospital Homburg/Saar, Germany ${ }^{5}$ Department of Biochemistry, University of Lausanne, Chemin des Boveresses 155, Epalinges, Switzerland

${ }^{6}$ Department of Clinical Pharmacology, University of Munich, Munich, Germany
\end{abstract}

\section{Correspondence to}

Dr Max Schnurr, Medizinische Klinik Innenstadt, University of Munich, Ziemssenstr. 1, D-80336 Munich, Germany; Max.Schnurr@med. uni-muenchen.de

CB and PD contributed equally to this work.

Revised 18 January 2010 Accepted 23 February 2010 Published Online First 4 May 2010

\begin{abstract}
Background The proinflammatory cytokines interleukin $1 \beta(I L-1 \beta)$ and IL-18 are central players in the pathogenesis of inflammatory bowel disease (IBD). In response to a variety of microbial components and crystalline substances, both cytokines are processed via the caspase-1-activating multiprotein complex, the NLRP3 inflammasome. Here, the role of the NLRP3 inflammasome in experimental colitis induced by dextran sodium sulfate (DSS) was examined.

Methods IL-1 $\beta$ production in response to DSS was studied in macrophages of wild-type, caspase- $1^{-1-}$, $\mathrm{NLRP}^{-/-}, \mathrm{ASC}^{-/-}$, cathepsin $\mathrm{B}^{-/-}$or cathepsin $\mathrm{L}^{-/-}$ mice. Colitis was induced in $\mathrm{C} 57 \mathrm{BL} / 6$ and $\mathrm{NLRP}^{-/-}$ mice by oral DSS administration. A clinical disease activity score was evaluated daily. Histological colitis severity and expression of cytokines were determined in colonic tissue.
\end{abstract}

Results Macrophages incubated with DSS in vitro secreted high levels of IL-1 $\beta$ in a caspase-1-dependent manner. IL-1 $\beta$ secretion was abrogated in macrophages lacking NLRP3, ASC or caspase-1, indicating that DSS activates caspase-1 via the NLRP3 inflammasome. Moreover, IL-1 $\beta$ secretion was dependent on phagocytosis, lysosomal maturation, cathepsin $\mathrm{B}$ and $\mathrm{L}$, and reactive oxygen species (ROS). After oral administration of DSS, NLRP3 ${ }^{-1-}$ mice developed a less severe colitis than wild-type mice and produced lower levels of proinflammatory cytokines in colonic tissue. Pharmacological inhibition of caspase- 1 with pralnacasan achieved a level of mucosal protection comparable with NLRP3 deficiency.

Conclusions The NLRP3 inflammasome was identified as a critical mechanism of intestinal inflammation in the DSS colitis model. The NLRP3 inflammasome may serve as a potential target for the development of novel therapeutics for patients with IBD.

\section{INTRODUCTION}

Human inflammatory bowel disease (IBD), the most important entities being ulcerative colitis and Crohn's disease, are chronic, relapsing and remitting inflammatory conditions that result from chronic dysregulation of the mucosal immune system in the gastrointestinal tract. ${ }^{1}$ The precise pathogenesis of IBD is still incompletely understood. However, it is now widely accepted that genetic and environmental factors are involved. Animal models of experimental colitis have been developed to investigate the molecular and cellular mechanisms leading to IBD, and these models are frequently used to develop and evaluate the efficacy

\section{Significance of this study}

What is known about this subject?

- The proinflammatory cytokines IL-1 $\beta$ and IL-18 are central players in the pathogenesis of IBD.

- Activation and secretion of the IL-1 cytokine family is regulated by the NLRP3 inflammasome, a caspase-1-activating multiprotein complex.

- Single nucleotide polymorphisms (SNPs) in the NLRP3 gene region and other inflammasomerelated genes are associated with susceptibility to Crohn's disease.

What are the new findings?

- Using the acute dextran sodium sulfate (DSS) colitis model we found that NLRP3-deficient mice were significantly protected from colitis.

- We identified DSS as a stimulus of macrophage NLRP3 inflammasome activation in vitro and in vivo.

- IL-1 $\beta$ secretion was dependent on phagocytosis of DSS macromolecules, lysosomal maturation, cathepsin $B$ and $L$, and reactive oxygen species (ROS).

\section{How might it impact on clinical practice in the foreseeable future? \\ - The NLRP3 inflammasome complex may serve as a potential target for the development of novel therapeutics for patients with IBD.}

of novel anti-inflammatory drugs. In the acute dextran sodium sulfate (DSS) colitis model mice are fed with DSS polymers in the drinking water and this induces a colitis characterised by diarrhoea, bloody faeces, weight loss and a histological picture of inflammation and ulceration as seen in human $\mathrm{IBD}^{2}$ As the acute inflammatory response is independent of $\mathrm{T}$ and $\mathrm{B}$ cells, ${ }^{3}$ the model is particularly useful to study the contribution of innate immune mechanisms in intestinal inflammation and, presumably due to toxic effects of DSS on the mucosa, epithelial barrier dysfunction.

Increased levels of proinflammatory cytokines, including interleukin-1 $\beta$ (IL-1 $\beta$ ), IL-6, IL-18 and tumour necrosis factor $\alpha$ (TNF $\alpha$ ), are detected in active IBD and correlate with the severity of inflammation. $^{4-6}$ IL- $1 \beta$ and TNF $\alpha$ have been shown to alter tight junctions and intestinal permeability. ${ }^{7}$ As epithelial barrier integrity is essential for blocking the access of microorganisms 
and toxins to underlying tissues, IL-1 $\beta$ is likely to be essential in the early phase of the inflammatory cascade leading to an inflamed colon. Indeed, enhanced levels of IL-1 $\beta$ are found in colonic mucosa and peritoneal macrophages in DSS-induced colitis and may hence represent an initial trigger of intestinal inflammation. ${ }^{8}$ IL-1 $\beta$ and IL-18 are activated by caspase- 1 , and studies with caspase- $1^{-/-}$mice strongly suggest that caspase- 1 plays a key role in DSS-induced colitis.

The NLR (nucleotide-binding domain and leucine-rich repeatcontaining) family comprises a group of intracellular pattern recognition receptors. NLRs have a leucine-rich repeat (LRR) that recognises diverse pathogen-associated molecular patterns. ${ }^{10}$ NOD2 and NLRP3 are two of the best characterised NLRs, and mutations of these receptors have been linked to Crohn's disease. NOD2 recognises the bacterial peptidoglycan-derived molecule muramyl dipeptide (MDP) and activates the nuclear factor- $\kappa \mathrm{B}$ $(\mathrm{NF}-\kappa \mathrm{B})$ pathway to induce an inflammatory response. Mutations of the NOD2 gene have been identified in individuals with Crohn's disease. ${ }^{11}{ }^{12}$ These polymorphisms have been linked to NF- $\kappa B$ activation and IL-1 $\beta$ secretion. ${ }^{13}$ However, the exact interaction of NOD2, NF- $\kappa \mathrm{B}$ and pro-IL-1 $\beta / \mathrm{IL}-1 \beta$ remains a matter of debate. ${ }^{14}$ NLRP3, also known as cryopyrin, forms an inflammasome with the adaptor molecule ASC and caspase- 1 to convert pro-IL-1 $\beta$ and pro-IL-18 into their active forms. Mutations in NLRP3 lead to chronic autoinflammatory syndromes. ${ }^{15}$ Single nucleotide polymorphisms (SNPs) in the NLRP3 gene region are associated with susceptibility to Crohn's disease. ${ }^{16}$ In addition, the combination of polymorphisms in NLRP3 and CARD8 was recently shown to be associated with Crohn's disease in Swedish male subjects. ${ }^{17}$

Recent studies have identified the autophagy protein Atg16L1 as a further susceptibility factor for Crohn's disease. ${ }^{18} 19$ Notably, Atg16L1 deficiency causes Toll/IL-1 receptor domaincontaining adaptor inducing interferon $\beta$ (TRIF)-dependent activation of caspase-1, and mice lacking Atg16L1 in haematopoietic cells are highly susceptible to DSS-induced acute colitis. ${ }^{20}$ Elevated systemic IL-1 $\beta$ and IL-18 levels in these mice correlated with severe mucosal inflammation, pointing towards an important role for deregulated caspase-1 activity in the pathogenesis of both IBD and DSS-induced colitis. ${ }^{9}$

In this study, we investigated the regulation of caspase- 1 activation in response to DSS in murine macrophages with genetic deletions of NLRP3 inflammasome components. In addition, the role of the NLRP3 inflammasome in intestinal inflammation was investigated using the acute DSS colitis model.

\section{METHODS}

\section{Cell culture and reagents}

Macrophage cell lines of wild-type (WT), caspase- $1^{-/-}$, NLRP3 ${ }^{-/-}$, $\mathrm{ASC}^{-/-}$, cathepsin $\mathrm{B}^{-/-}$, cathepsin $\mathrm{L}^{-/-}$and $\mathrm{IPAF}^{-/-}$mice were generated as described. ${ }^{21}$ Cells were cultured in Dulbecco's modified Eagle's medium (DMEM) high glucose supplemented with 1\% L-glutamine (all PAA, Pasching, Austria), 10\% fetal calf serum (FCS; GIBCO, Karlsruhe, Germany) and $10 \mu \mathrm{g} / \mathrm{ml}$ ciprofloxacin (Hexal, Holzkirchen, Germany). Human THP-1 cells were cultivated in RPMI supplemented with 10\% FCS, 1\% Na-pyruvate and $10 \mu \mathrm{g} / \mathrm{ml}$ ciprofloxacin, and differentiated with phorbol 12-myristate 13-acetate (PMA; $5 \mathrm{nM}$ ) 3 h before DSS stimulation. Primary human macrophages were generated from adherent peripheral blood mononuclear cells (PBMCs) of healthy donors and cultivated in RPMI with $2 \% \mathrm{AB}$ serum (Lonza, Verviers, Belgium), 1\% L-glutamine, $100 \mathrm{U} / \mathrm{ml}$ penicillin, $0.1 \mathrm{mg} / \mathrm{ml}$ streptomycin (PAA, Pasching, Austria) in the presence of $1000 \mathrm{U} / \mathrm{ml}$ recombinant human granulocyte-stimulating factor (rhGM-CSF;
Berlex, Richmond, California, USA). The caspase-1 inhibitor z-YVAD-fmk, nigericin, cytochalasin D and bafilomycin A1 were purchased from Calbiochem (Darmstadt, Germany). Poly(dA:dT) sodium salt, $N$-acetyl-L-cysteine (NAC), ammonium pyrrolidinedithiocarbamate (APDC) and dextranase were from Sigma-Aldrich (Munich, Germany). All DSS reagents were from MP Biomedicals (Illkirch, France). Ultra pure lipolysaccharide (LPS) K12 and acridine orange were from Invitrogen (Toulouse, France). The caspase-1 inhibitor pralnacasan was provided by Sanofi-Aventis (Frankfurt, Germany). Cremophor EL was purchased from BASF (Ludwigshafen, Germany).

\section{Speck formation assay}

Assembly of the ASC-containing NLRP3 inflammasome was studied using macrophages that stably express the fusion protein ASC-cyan fluorescent protein (CFP). Cells were plated at a density of $10^{6}$ cells/well, primed with $10 \mathrm{ng} / \mathrm{ml}$ LPS for $2 \mathrm{~h}$ and incubated with DSS for $24 \mathrm{~h}$. The cells were washed and ASC-CFP speck formation was analysed by fluorescence microscopy (Zeiss, Jena, Germany) and ImageJ software (NIH, Bethesda, Maryland, USA) for digitally counting specks per high power field (HPF).

\section{Flow cytometry}

For the analysis of lysosomal damage macrophages were plated into 24-well culture dishes and stimulated for $24 \mathrm{~h}$ with DSS. The cells were washed and incubated with $1 \mu \mathrm{g} / \mathrm{ml}$ acridine orange for $15 \mathrm{~min}$ for staining of lysosomes. Cells were washed twice and fluorescence intensity was analysed at $600-650 \mathrm{~nm}$ emission wavelength with a FACSCanto II (BD Biosciences San Diego, California, USA). Data analysis was performed using FlowJo software (Tree Star, Ashland, Oregon, USA).

\section{Mice}

$\mathrm{NLRP3}^{-/-}$mice ${ }^{22}$ were bred at the University of Munich and used for experiments between the ages of 8 and 16 weeks. Agematched WT controls were purchased from Harlan Winkelmann (Borchen, Germany). Mice were fed standard mice chow pellets, had access to tap water supplied in bottles, and were acclimatised at least 7 days before they entered into experiments. All experiments were approved by the regional animal study committee and are in agreement with the guidelines for the proper use of animals in biomedical research.

\section{Induction of colitis and treatment}

Colitis was induced in C57BL/6 and NLRP3 ${ }^{-/-}$mice with $2 \%$ DSS (molecular weight $=40 \mathrm{kDa}$ ) dissolved in drinking water given ad libitum (days 1-9) as described. ${ }^{2}$ Control mice were given tap water. The caspase-1 inhibitor pralnacasan was dissolved in $25 \%$ Cremophor EL solution and was filtered through syringe filters $(0.2 \mu \mathrm{m})$. The substance was administered intraperitoneally at a dosage of $50 \mathrm{mg} / \mathrm{kg}$ body weight twice daily. Control animals received Cremophor EL intraperitoneally twice daily.

\section{Clinical score and histological analysis}

Body weight, the presence of occult or gross blood per rectum, and stool consistency were determined by two investigators blinded to the treatment groups. A scoring system was applied to assess diarrhoea and the presence of occult or overt blood in the stool. ${ }^{23}$ Changes of body weight are indicated as loss of baseline body weight as a percentage. Postmortem, the colon was removed and pieces of colonic tissue were used for ex vivo analysis. For histology, rings of the transverse part of the colon were fixed in $4 \%$ buffered formalin and embedded in paraffin. Sections 
were stained with $\mathrm{H} \& \mathrm{E}$ according to standard protocols. Histological scoring was performed in a blinded way by a pathologist (HAL). Focally increased numbers of inflammatory cells in the lamina propria were scored as 1 , confluence of inflammatory cells extending into the submucosa as 2 and transmural extension of the infiltrate as 3 . For tissue damage, discrete lymphoepithelial lesions were scored as 1 , mucosal erosions as 2 , and extensive mucosal damage and/or extension through deeper structures of the bowel wall as 3 . The two equally weighted subscores (cell infiltration and tissue damage) were added and the combined histological colitis severity score ranged from 0 to 6 .

\section{Ex vivo analysis of colonic cytokines}

Strips of colon were mechanically crushed, vortexed in $200 \mu \mathrm{l}$ of Tissue Protein Extraction Reagent (Pierce, Rockford, USA) for $1 \mathrm{~min}$ and shock frozen in liquid nitrogen. The homogenate was centrifuged at $10000 \mathrm{~g}$ at $4^{\circ} \mathrm{C}$ for $15 \mathrm{~min}$. The amount of total extracted protein was determined by Bradford analysis using the BioRad Protein Assay (BioRad, Munich, Germany). The amount of IFN $\gamma$, IL-1 $\beta$ and TNF $\alpha$ in the colon homogenate was quantified by ELISA (BD Biosciences Pharmingen, San Diego, California, USA).

\section{mRNA extraction and reverse transcription-PCR (RT-PCR)}

Colonic tissue was cleaned in phosphate-buffered saline (PBS), snap-frozen in liquid nitrogen and stored at $-70^{\circ} \mathrm{C}$. Total cellular RNA was isolated by homogenising tissue with an Ultra Turrax instrument (Janke und Kunkel, Staufen im Breisgau, Germany) and using the Roche Total RNA Tissue Extraction Kit (Roche, Mannheim, Germany). The yield and purity of the RNA were determined by spectroscopic analysis and the concentration of total RNA was equilibrated. For reverse transcription, M-MLV reverse transcriptase (Gibco Life Technologies, Paisley, UK), RNase inhibitor (Roche), oligo(dT) primer for cDNA synthesis (Roche) and dNTP (Promega, Madison, Wisconsin, USA) were used. A Light Cycler Instrument (Roche) and the Light Cycler Fast Start DNA Master SYBR Green I Kit (Roche) were used for real-time PCR, according to the manufacturer's recommendations. Primers for murine glyceraldehyde phosphate dehydrogenase (GAPDH) and IP-10 (CXCL-10) were purchased as Light Cycler Primer Sets including standard DNA from Search-LC (Heidelberg, Germany). The number of copies in each sample was correlated with the number of GAPDH copies.

\section{Isolation of peritoneal macrophages}

Mice were sacrified by cervical dislocation under isoflurane anaesthesia, injected with $10 \mathrm{ml}$ of PBS intraperitoneally and, after shaking, peritoneal lavage was performed. Collected peritoneal lavage fluid was centrifuged and erythrocytes in the cell pellet were lysed using BD Pharm Lyse lysing buffer (BD Bioscience). The remaining cells were plated into culture dishes overnight and adherent cells were used for cytokine assays.

\section{ELISA}

Primary macrophages and cell lines were seeded into 96-well plates at a density of $2 \times 10^{5}$ cells per well. After LPS priming for $1 \mathrm{~h}$, cells were stimulated with the indicated amounts of DSS for $24 \mathrm{~h}$. Cell culture supernatant or colon homogenates were used for ELISAs (BD Bioscience), which were performed according to the manufacturer's protocol.

\section{SDS-PAGE and western blotting}

Cell culture supernatants of $10^{6}$ cells or $65 \mu \mathrm{g}$ of whole protein from colon homogenate were dissolved in Laemmli buffer
(BioRad) and separated using a 15\% acrylamide-bisacrylamide gel. Proteins were blotted onto a $0.45 \mu \mathrm{m}$ polyvinylidene fluoride (PVDF) membrane (Millipore, Schwalbach, Germany). Primary anti-caspase-1 (rabbit, antimouse, Santa Cruz) and anti-IL-1 $\beta$ (R\&D Systems, Wiesbaden-Nordenstadt, Germany) were applied at 1:500 and horseradish peroxidase (HRP)-coupled B-actin (loading control) was used at 1:3000. Immunoglobulin G (IgG) antigoat-HRP (Santa Cruz) was diluted 1:3000 and Amersham ECL ${ }^{\text {TM }}$ (GE Healthcare, Munich, Germany) served for visualisation via chemoluminescence.

\section{Statistical analysis}

Data are expressed as means \pm SEM. Statistical significance of differences between treatment and control groups was determined by Student t test. Differences were considered statistically significant at $\mathrm{p}<0.05$.

\section{RESULTS \\ DSS induces caspase-1-dependent IL-1 $\beta$ processing in murine macrophages}

Release of active IL- $1 \beta$ is mediated by a two-step process initiated by transcriptional induction of pro-IL-1 $\beta$, for example by a Toll-like receptor (TLR) stimulus, followed by caspase-1mediated cleavage. DSS, a polyanionic derivate of sulfated high molecular weight dextrane, induces the release of IL-1 $\beta$ from murine macrophages. ${ }^{8}$ To investigate the mechanism of IL- $1 \beta$ release, we incubated a murine macrophage cell line with DSS for $24 \mathrm{~h}$ with or without prior LPS priming. In the absence of LPS priming, DSS did not induce a notable release of IL-1 $\beta$. However, LPS-primed macrophages strongly responded to the addition of DSS in a dose-dependent manner (figure 1A). The requirement of LPS priming for enhanced IL-1 $\beta$ synthesis was further supported by data obtained from a macrophage cell line of mice with combined MyD88 and TRIF deficiency. These cells failed to secrete IL-1 $\beta$ in response to either DSS or nigericin, a potassium ionophore activating caspase-1, but responded to poly $(\mathrm{dA}: \mathrm{dT})$, which induces IL-1 $\beta$ in a TLR-independent manner (figure $1 \mathrm{~B})$. To demonstrate the role of caspase- 1 in DSSmediated IL-1 $\beta$ release, we incubated the macrophages with the caspase-1 inhibitor z-YVAD-fmk and found that this completely abolished the release of IL-1 $\beta$ (figure $1 \mathrm{C}$ ). Similar results were obtained using primary peritoneal macrophages from WT mice (figure 1D) as well as primary human macrophages and THP-1 cells (Supplementary figure 1). To confirm that IL-1 $\beta$ is released in its active form, we performed western blotting of IL-1 $\beta$ p17 and caspase- 1 p10, which were both present in supernatants of DSS- or nigericin-stimulated macrophages (figure 1E). To determine whether intact DSS macromolecules were required for caspase-1 activation, we digested DSS (40 kDa) with dextranase, which cleaves 1,6-glycosylic bounds of isomaltose, before macrophage stimulation. Dextranase almost completely inhibited the release of IL-1 $\beta$ (figure $1 F$ ). Incubating macrophages with DSS molecules ranging from 8 to $1400 \mathrm{kDa}$ revealed a positive correlation between IL-1 $\beta$ release and DSS molecular weight (figure 1G), suggesting that IL-1 $\beta$ secretion is induced only by intact DSS macromolecules.

\section{DSS induces NLRP3 inflammasome activation}

The NLR protein NLRP3 can form an inflammasome complex with the adaptor molecule ASC and caspase- 1 in response to various stimuli. ${ }^{24}$ We speculated that caspase- 1 activation by DSS is mediated by the NLRP3 inflammasome. It has been shown that NLRP3 inflammasome activation is dependent 

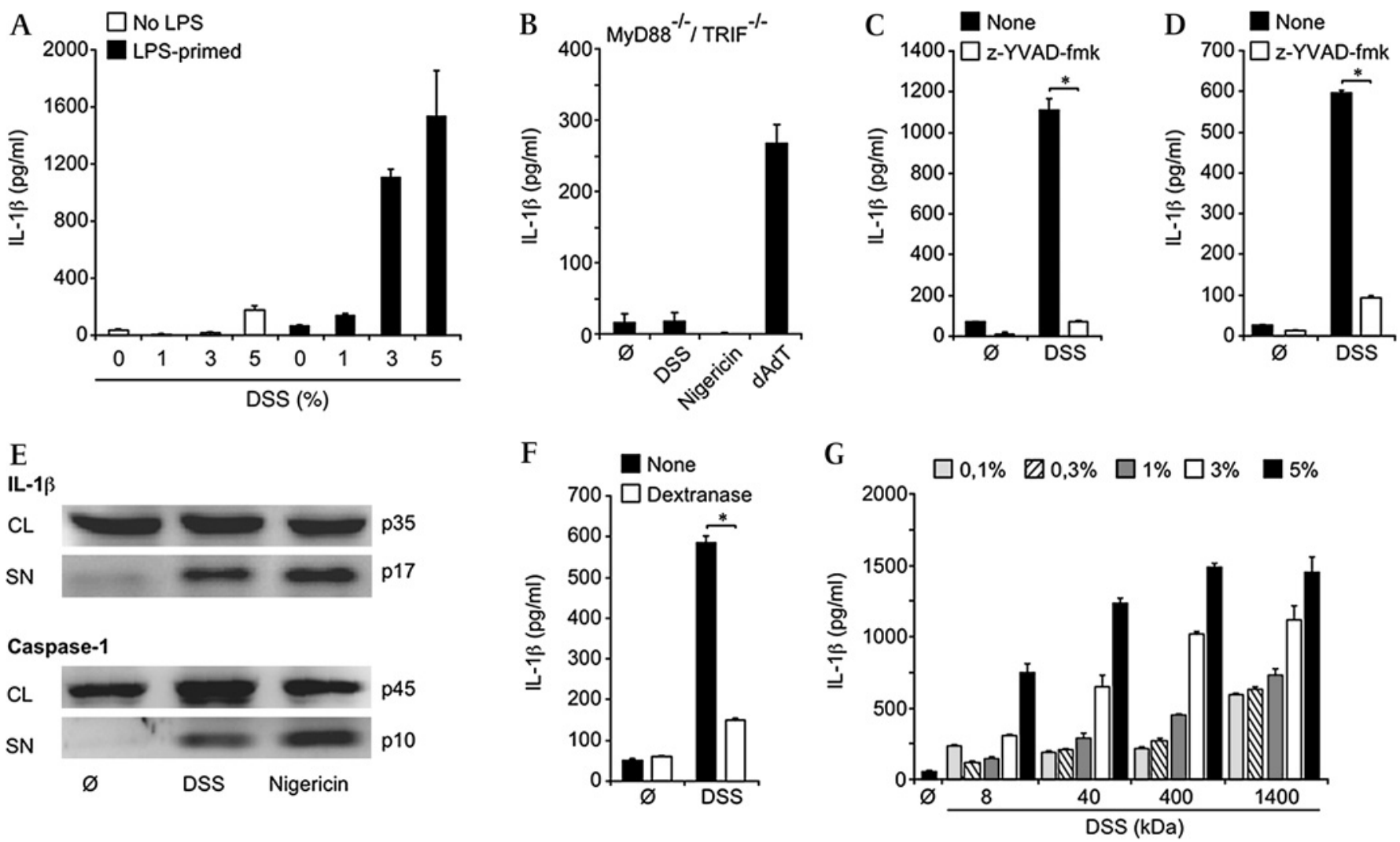

Figure 1 Dextran sodium sulfate (DSS) induces caspase-1-mediated interleukin $1 \beta$ (IL-1 $\beta$ ) release from murine macrophages. (A) Macrophages were treated with increasing concentrations of DSS in the absence or presence of lipopolysaccharide (LPS) priming. IL-1 $\beta$ was determined in the supernatant by ELISA. (B) Absence of IL-1 $\beta$ release of LPS-primed MyD88/TRIF-deficient macrophages in response to DSS or nigericin. Transfected dAdT served as a MyD88/TRIF-independent stimulus. (C) Influence of the caspase-1 inhibitor z-YVAD-fmk (10 $\mu \mathrm{M})$ on IL-1 $\beta$ release by a macrophage cell line and (D) by primary macrophages. (E) Western blot analysis of IL-1 $\beta$ p35 and caspase-1 p45 in cell lysates (CL) and of bioactive IL-1 $\beta$ p17 and caspase-1 p10 in supernatants (SN) of DSS- or nigericin-stimulated, LPS-primed macrophages. (F) Influence of dextranase treatment of DSS on IL-1 $\beta$ release by macrophages. (G) Influence of DSS molecule size (8 to $1400 \mathrm{kDa}$ ) on IL-1 $\beta$ release. Shown are representative data as means \pm SEM ( $\mathrm{n}=3$ independent experiments). ${ }^{*} \mathrm{p}<0.05$. TRIF, Toll/IL-1 receptor domain-containing adaptor inducing interferon $\beta$.

on $\mathrm{K}^{+}$efflux. $^{25}$ To assess the role of NLRP3 in DSS-induced caspase- 1 activation, we blocked $\mathrm{K}^{+}$efflux by the addition of high concentrations of $\mathrm{KCl}$ to the extracellular medium and found that this completely inhibited IL- $1 \beta$ release (figure $2 \mathrm{~A}$ ). In addition, macrophages lacking NLRP3, ASC or caspase-1 were devoid of IL-1 $\beta$ secretion in response to DSS exposure (figure $2 \mathrm{~B}$ ). Consistent with previous observations, ${ }^{21}$ response to transfected poly(dA:dT) was NLRP3 independent and ASC dependent. In contrast, macrophages from IPAF ${ }^{-1-}$ mice (IPAF is an NLRP3-independent, caspase-1-recruiting inflammasome) showed no defect in IL-1 $\beta$ secretion (figure $2 \mathrm{C}$ ). The requirement of NLRP3 for IL-1 $\beta$ release was further supported by studying primary peritoneal macrophages from $\mathrm{NLRP}^{-/-}$mice (figure 2D). NLRP3 activation triggers the formation of a large assembly of ASC, which rapidly activates caspase-1. To analyse the recruitment of ASC, we used macrophages stably expressing ASC fused to the fluorescent protein $\mathrm{CFP}^{26}$ NLRP3-ASC assembly results in the formation of fluorescent specks, which can be visualised by fluorescence microscopy. DSS induced speck formation in a dose-dependent manner (figure 2E). We could rule out that DSS, either directly or indirectly, induces NLRP3 inflammasome activation via the P2X7 receptor ${ }^{27}$ by demonstrating that IL- $1 \beta$ secretion in response to DSS was unimpaired in macrophages lacking P2X7 (figure 2F). Together, these findings indicate that DSS activates the NLRP3-ASC complex, leading to the activation of caspase- 1 and subsequent cleavage of pro-IL-1 $\beta$ into the mature, secreted form.

\section{NLRP3 inflammasome activation in response to DSS is dependent on lysosomal maturation and reactive oxygen species (ROS)}

Phagocytosis of particulate danger-associated molecular patterns has been shown to activate the NLRP3 inflammasome via lysosomal destabilisation. ${ }^{21}$ To characterise the mechanisms of NLRP3 inflammasome activation in response to DSS further, we used pharmacological inhibitors to block pathways of lysosome formation and function. The role of phagosome formation in caspase- 1 activation was studied by incubating macrophages prior to DSS stimulation with cytochalasin D, which inhibits phagocytosis by disrupting actin filaments. Cytochalasin D completely abrogated IL-1 $\beta$ secretion in response to DSS, whereas the response to the potassium ionophore nigericin was unaffected (figure $3 \mathrm{~A}$ ). Moreover, blocking lysosomal acidification with bafilomycin A1, an inhibitor of the vacuolar $\mathrm{H}^{+}$ATPase, completely inhibited DSS-mediated IL- $1 \beta$ release (figure $3 \mathrm{~A}$ ). These findings suggest a critical role for functional lysosomes in DSS-mediated NLRP3 activation. Experiments using specific inhibitors of cathepsin B have shown a link between lysosomal cysteine proteinases and NLRP3 inflammasome activation for crystals and influenza virus. ${ }^{21} 28$ To assess whether this mechanism is operative during DSS-mediated NLRP3 inflammasome 
Figure 2 Interleukin $1 \beta$ (IL-1 $\beta)$ processing in response to dextran sodium sulfate (DSS) is mediated by the NLRP3 inflammasome. (A) Macrophages were primed with lipopolysaccharide (LPS) and incubated with $3 \%$ DSS in the presence or absence of high concentrations of $\mathrm{KCl}(130 \mathrm{mM})$. IL-1 $\beta$ was determined in the supernatant by ELISA. (B) LPS-primed macrophage cell lines from wildtype (WT), $\mathrm{NLRP3}^{-/-}, \mathrm{ASC}^{-/-}$or caspase- $1^{-1-}$ mice were incubated with $3 \%$ DSS. Incubation with dAdT served as a NLRP3-independent, but ASCdependent stimulus. (C) IL-1 $\beta$ secretion by macrophages deficient in the IPAF inflammasome. (D) IL-1 $\beta$ secretion in response to $3 \%$ DSS by primary macrophages from WT and NLRP3 ${ }^{-1-}$ mice. (E) Recruitment of ASC to the inflammasome was visualised using macrophages expressing an ASC-cyan fluorescent protein (CFP) fusion protein by fluorescence microscopy. The numbers of specks per high power field (HPF) were counted. (F) IL-1 $\beta$ secretion by macrophages deficient in the P2X7 receptor. Shown are representative data as means \pm SEM ( $n=3$ independent experiments). ${ }^{*} \mathrm{p}<0.05$. activation, we compared IL- $1 \beta$ release of the WT with that of cathepsin B- and cathepsin L-deficient macrophages. IL-1 $\beta$ secretion in DSS-treated macrophages was significantly reduced in cathepsin B- and, to a lesser extent, in cathepsin L-deficient macrophages (figure $3 \mathrm{~B}$ ). Thus, lysosomal proteases appear to be involved in DSS-induced NLRP3 inflammasome activation. We next assessed whether DSS leads to lysosomal damage, as has been proposed for crystalline structures. ${ }^{21}$ We made use of the staining properties of acridine orange, a dye with green fluorescence in its monomeric state and red fluorescence when forming dimers in acidic compartments, the red fluorescence intensity correlating with the cytoplasmic number of lysosomes. Indeed, DSS induced a loss of red fluorescence of macrophages stained with acridine orange at similar levels to those previously reported for crystals, ${ }^{21}$ suggesting lysosomal damage (figure 3C). Taken together, these findings point towards a DSS-mediated phagosomal destabilisation, leading to the release of phagosomal contents into the cytosol where they are sensed by the NLRP3 inflammasome.

NLRP3 inflammasome activation has been linked with ROS generation in response to various stimuli. ${ }^{28} 29$ DSS has previously been shown to stimulate macrophage ROS production. ${ }^{30}$ To investigate the role of ROS in DSS-induced IL-1 $\beta$ release, we treated macrophages with the ROS inhibitors $N$-acetylcysteine (NAC) and ammonium pyrrolidinedithiocarbamate (APDC). Both inhibitors significantly reduced IL-1 $\beta$ secretion (figure $3 D$ ). These data suggest that ROS contribute to DSS-induced NLRP3 inflammasome activation.
Figure 3 Activation of the NLRP3 inflammasome by dextran sodium sulfate (DSS) requires lysosomal maturation and reactive oxygen species (ROS). (A) Macrophages were incubated with cytochalasin $\mathrm{D}(2 \mu \mathrm{M})$ or bafilomycin $\mathrm{A} 1$ (20 $\mathrm{nM})$ before treatment with $3 \%$ DSS. The potassium ionophore nigericin served as positive control. Interleukin $1 \beta$ (IL-1 $\beta$ ) was determined by ELISA. (B) Macrophage cell lines from wild-type (WT), cathepsin $\mathrm{B}^{-/-}$or cathepsin $\mathrm{L}^{-/-}$mice were primed with lipopolysaccharide (LPS) and incubated with DSS. (C) Phagosomes of DSS-treated macrophages were stained with the fluorochrome acridine orange. Loss of fluorescence, which correlates with reduced numbers of lysosomes, was analysed by fluorescence-activated cell sorting. (D) Influence of the ROS inhibitors, ammonium pyrrolidinedithiocarbamate (APDC) and $\mathrm{N}$-acetyl-L-cysteine (NAC), on DSS-induced IL-1 $\beta$ production. Nigericin served as a positive control. Shown are representative data as means \pm SEM ( $n=3$ independent experiments). ${ }^{*} \mathrm{p}<0.05$.
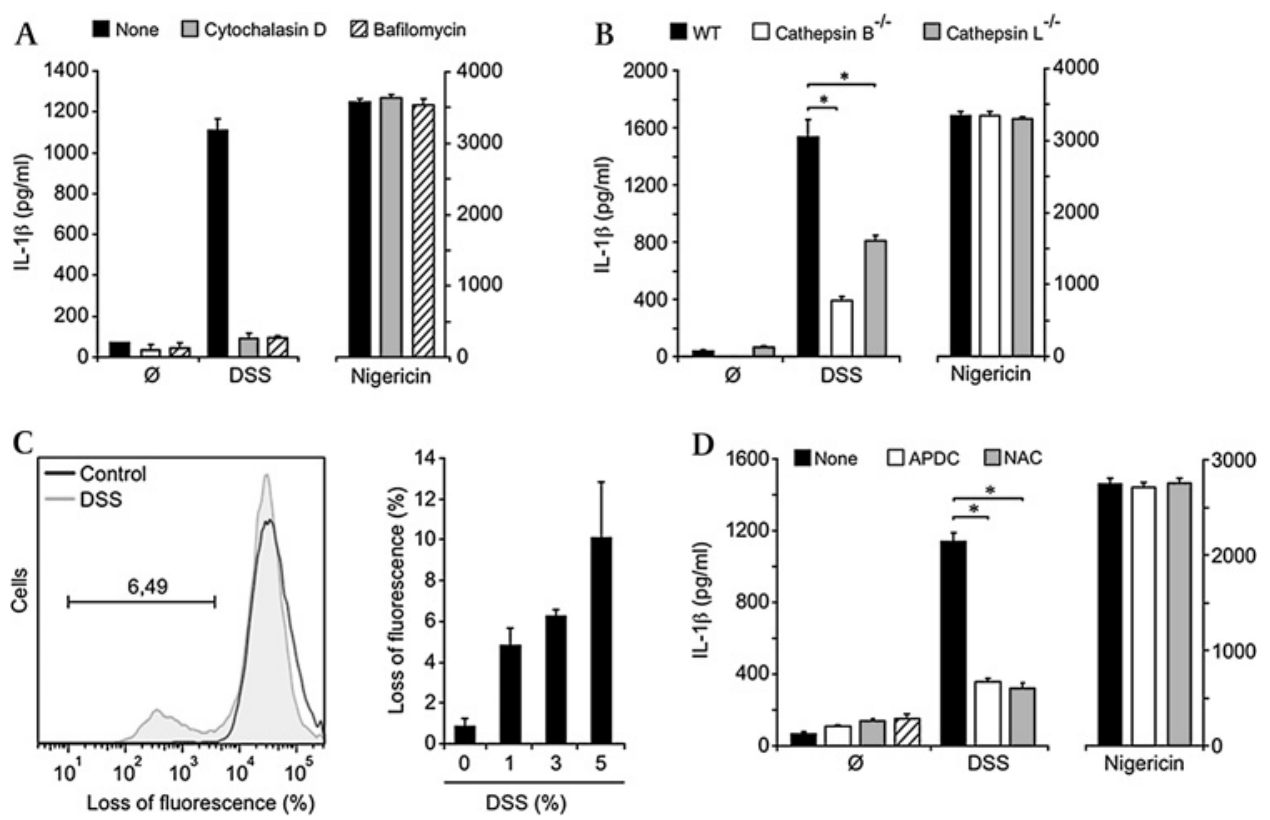


\section{Colitis severity and inflammatory response in the DSS model depend on NLRP3 signalling}

We next investigated the role of the NLRP3 inflammasome in DSS-mediated intestinal inflammation in vivo. WT and $\mathrm{NLRP}^{-/-}$mice received $2 \%$ DSS in their drinking water for a period of 9 days. Clinical parameters, including body weight, the presence of occult or gross blood per rectum and stool consistency, were determined daily. NLRP3 ${ }^{-/-}$mice were significantly protected from DSS-induced colitis, showing reduced loss of body weight and haematochezia (figure 4A). Of note, four out of 15 WT mice had died due to colitis by day 9, whereas all 15 $\mathrm{NLRP}^{-1-}$ mice survived. Histological analysis of colonic tissue obtained on day 6 revealed less severe mucosal infiltration by inflammatory cells and reduced tissue damage in NLRP3 ${ }^{-1-}$ mice, translating into a significantly improved histological colitis severity score (figure $4 \mathrm{~B}$ and Supplementary figure 2). On day 9 , histology showed severe intestinal inflammation with destruction of the epithelial layer leading to identical histology scores in WT and NLRP3 ${ }^{-/}$mice, despite the reduced clinical colitis scores in NLRP3 ${ }^{-1-}$ mice. These findings indicate that the NLRP inflammasome plays a more critical role during the early phase of colitis induction, but cannot prevent colitis progression after prolonged DSS exposure. As caspase- 1 activity is regulated by NLRP3, inhibition of caspase-1 could be an effective novel treatment strategy for IBD. In line with this notion, we have previously reported that pharmacological inhibition of caspase- 1 with pralnacasan is effective in the treatment of DSS-induced experimental colitis. ${ }^{23}$ Treatment of mice with pralnacasan, at a dose that had been optimal in our previous study, improved clinical parameters, such as body weight, stool consistency and haematochezia (figure 4C). Indeed, the magnitude of the therapeutic benefit was similar to the protective effect observed in $\mathrm{NLRP}^{-/-}$mice, providing further evidence that caspase- 1 activation via the NLRP3 inflammasome is a major pathological mechanism in DSS-induced colitis.

Proinflammatory cytokines, including IL-1 $\beta, \mathrm{TNF} \alpha$ and IFN $\gamma$, are elevated in colonic tissue in humans and in the acute DSS colitis model. To assess the influence of the NLRP3 inflammasome on the intestinal inflammatory response, we first analysed IL-1 $\beta$ production of peritoneal macrophages isolated from mice receiving DSS in their drinking water. Macrophages of WT mice spontaneously released low levels of IL-1 $\beta$, which was strongly enhanced by DSS feeding. In contrast, macrophages of $\mathrm{NLRP3}^{-/-}$mice secreted no detectable levels of IL-1 $\beta$ in the absence of DSS feeding and only low levels in response to DSS (figure 5A). Furthermore, levels of TNF $\alpha$, IFN $\gamma$ and IP-10 in colonic homogenates were elevated in WT but not in NLRP3 ${ }^{-/-}$ mice in response to 6 days of DSS feeding (figure $5 \mathrm{~B}$ ). Interestingly, levels of these cytokines were also reduced in NLRP3 ${ }^{-1-}$ mice receiving tap water only, indicating that NLRP3 might also play a role in physiological intestinal homeostasis. Of interest, IL-1 $\beta$ levels in colonic homogenates measured by ELISA did not differ significantly between WT and NLRP3 ${ }^{-1-}$ mice. As the ELISA does not discriminate between pro-IL-1 $\beta$ and active IL-1 $\beta$, we performed western blot analysis for cleaved IL-1 $\beta$ ( $p 17$ ) and found high levels of cleaved IL-1 $\beta$ in the colon of WT mice receiving DSS, but not in that of $\mathrm{NLRP3}^{-/-}$mice (figure 5C).
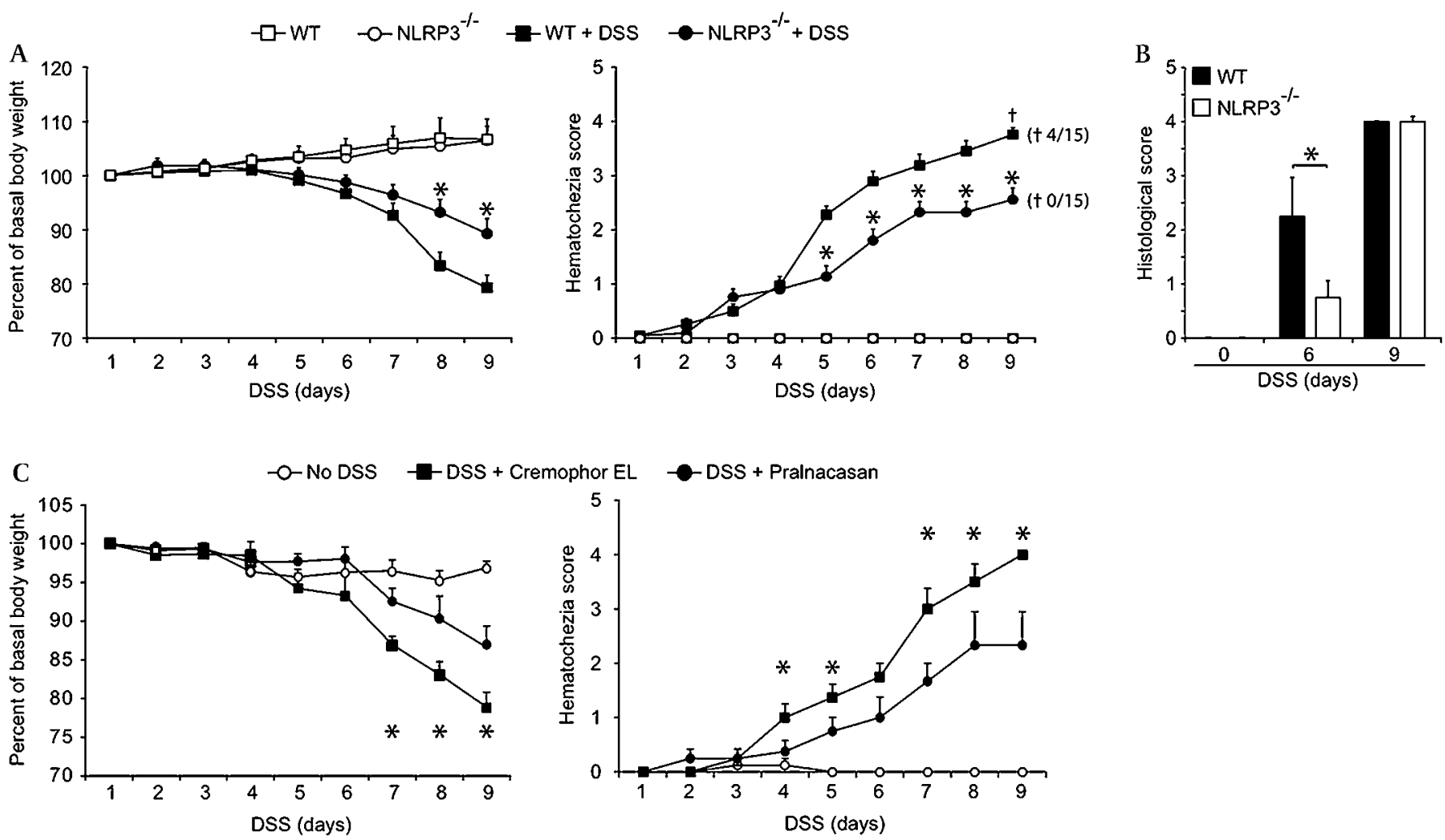

Figure 4 NLRP3 deficiency and caspase-1 inhibition protects mice from dextran sodium sulfate (DSS)-induced colitis. (A) Loss of basal body weight and haematochezia score of wild-type (WT) and NLRP3 ${ }^{-1-}$ mice ( $n=15$ per group) receiving $2 \%$ DSS or tap water for 9 days. $\dagger$ indicates death rate. (B) Histological score of distal colon sections of WT and NLRP3 ${ }^{-/-}$mice receiving DSS or tap water on day 6 and day 9 of DSS feeding. (C) Loss of basal body weight and haematochezia score of WT mice treated with tap water or $2 \%$ DSS orally ad libitum in the presence or absence of the caspase1 -inhibitor pralnacasan $(50 \mathrm{mg} / \mathrm{kg}$ intraperitoneally) or with Cremophor EL (vehicle) alone. Data are presented as means \pm SEM ( $\mathrm{n}=2$ independent experiments). ${ }^{*} \mathrm{p}<0.05$. 

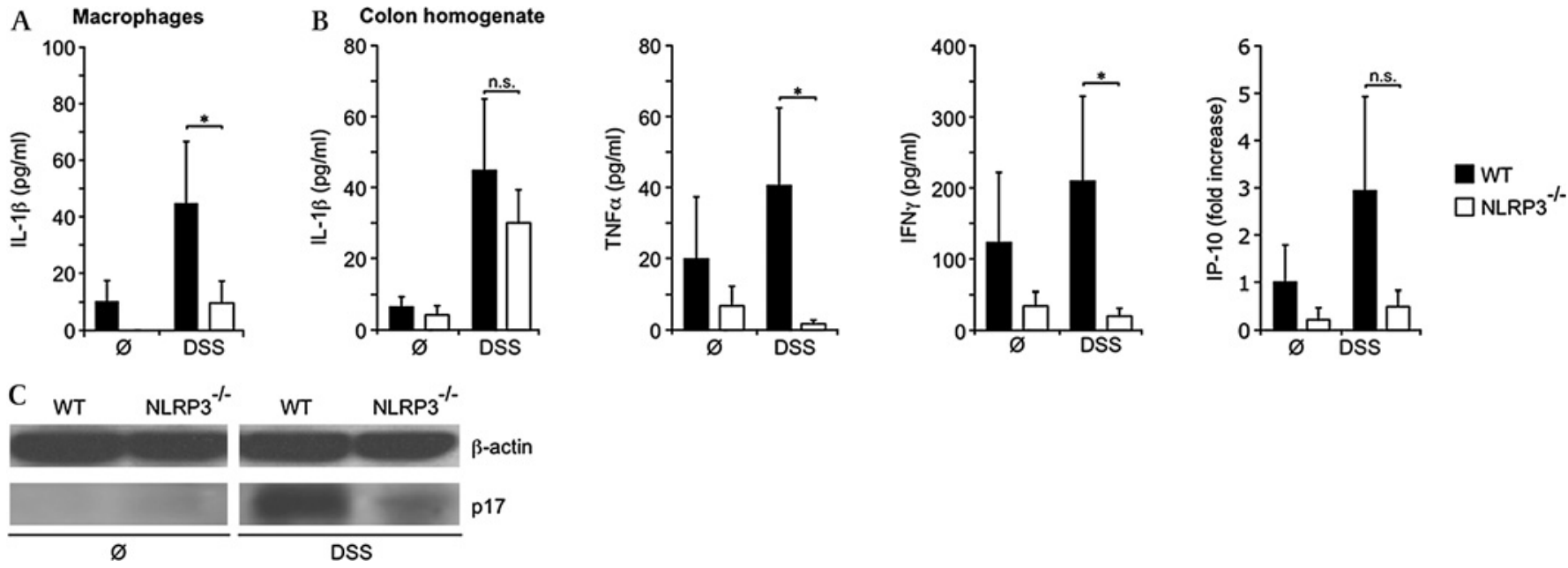

Figure 5 Reduced inflammatory responses in NLRP3 ${ }^{-1-}$ mice in response to dextran sodium sulfate (DSS). (A) Peritoneal macrophages of wild-type (WT) and NLRP3 ${ }^{-1-}$ mice ( $n=5$ per group) treated with $2 \%$ DSS for 9 days or with tap water were obtained by lavage, and spontaneous release of interleukin $1 \beta$ (IL-1 $\beta$ ) was determined in culture supernatant by ELISA. (B) Cytokine levels in colonic homogenate of WT and NLRP3 ${ }^{-1-}$ mice. Levels of IL-1 $\beta$, tumour necrosis factor $\alpha$ (TNF $\alpha$ ) and interferon $\gamma$ (IFN $\gamma$ ) were analysed by ELISA. IP-10 expression was assessed by quantitative reverse transcription-PCR. Data are presented as means \pm SEM ( $n=2$ independent experiments). ${ }^{*} p<0.05$. (C) Western blots of IL-1 $\beta$ ( $\left.p 17\right)$ in colonic homogenate of DSS-treated WT and NLRP3 ${ }^{-1-}$ mice.

\section{DISCUSSION}

Data from human specimens and from murine colitis models indicate that excessive expression of IL- $1 \beta$ and IL-18 plays a key role in the pathogenesis of IBD. ${ }^{5}{ }^{31}$ Furthermore, caspase-1, which regulates the secretion of biologically active IL-1 $\beta$ and IL-18, has been identified as a central mediator of DSS-induced colitis. ${ }^{9}$ In the present study, we provide evidence that DSS induces caspase-1 activation via the NLRP3 inflammasome in macrophages and that $\mathrm{NLRP}^{-1-}$ mice are significantly protected from DSS-induced colitis, developing a decreased clinical and histological colitis severity and dramatically reduced levels of proinflammatory cytokines in the colonic tissue. This protective effect was most pronounced at an early time point of the disease, indicating a critical role for the NLRP3 inflammasome at the initiation of the inflammatory process. Together, these results strongly argue for a role for the NLRP3 inflammasome in DSS-induced colitis. Interestingly, the clinical colitis severity parameters in NLRP3 ${ }^{-1-}$ mice were comparable with those achieved by inhibition of caspase- 1 with pralnacasan in WT mice, indicating that pharmacological intervention in the NLRP3 inflammasome complex may have therapeutic potential in the treatment of IBD. ${ }^{23}$

The precise mechanism of NLRP3 inflammasome activation is incompletely understood. The data obtained in the present study demonstrate that IL-1 $\beta$ release by macrophages in response to DSS requires lysosomal maturation, the lysosomal proteases cathepsin B and cathepsin L, loss of lysosomal integrity as well as ROS production. These mechanisms resemble those previously described for other NLRP3 stimuli, such as monosodium urate, asbestos, silica crystals and influenza virus. $^{21} 2829$ Assays measuring IL-1 $\beta$ production by DSSstimulated macrophages could be exploited for high-throughput screening of new anti-inflammatory drugs targeting the NLRP3 inflammasome for the treatment of IBD.

In what respect can these findings contribute to our understanding of human IBD? Genetic studies provide evidence for an association of IBD with mutations of NLRP3 inflammasome components. Villani and co-workers found that susceptibility to Crohn's disease is associated with polymorphisms located in a predicted regulatory region on chromosome 1q44 downstream of NLRP3. ${ }^{16}$ Interestingly, these polymorphisms correlated with impairment of IL-1 $\beta$ processing of PBMCs in response to LPS, indicating that NLRP3-mediated caspase- 1 activation may play a protective role in IBD, for example in the defence of intestinal microbial pathogens. On the other hand, McGovern and coworkers detected an association between IBD and a polymorphism of CARD8 (C10X), ${ }^{32}$ even though this could not be confirmed by others. ${ }^{33}{ }^{34}$ CARD8 is a binding partner of NLRP3 and as such a component of the NLRP3 inflammasome. ${ }^{35}$ It is known to suppress NF-KB activity and to regulate caspase- 1 activation. ${ }^{36}{ }^{37}$ Similarly to mutations in NOD2, CARD8 mutations might lead to perturbations in the NF- $\kappa B$ pathway, finally leading to upregulation of pro-IL-1 $\beta$ and pro-IL-18 in lamina propria macrophages. In the same model, an additional gain-of-function mutation of NLRP3 could lead to an overactivated caspase- 1 , cleaving pro-IL-1 $\beta$ and pro-IL-18 into their biologically active forms. In support of this hypothesis, Schoultz and co-workers have recently reported that combined NLRP3 and CARD8 mutations increase the susceptibility to Crohn's disease in a cohort of Swedish men. ${ }^{17}$

This 'two-hit' model of caspase-1 activation leading to the secretion of IL-1 $\beta$ and IL-18 in human IBD appears to be closely mirrored in the DSS colitis model in mice. Initially, DSS exerts a direct toxic effect on the epithelial barrier, hence allowing bacteria to stimulate lamina propria macrophages via TLRs and the NF- $\mathrm{BB}$ pathway, which leads to enhanced transcription of pro-IL-1 $\beta$ and pro-IL-18. ${ }^{38}$ In a second step, DSS induces caspase-1 activation via the NLRP3 inflammasome complex, cleaving IL-1 $\beta$ and IL-18 into their biologically active forms, which initiates an intestinal inflammatory cascade. Our data may thus help to understand why DSS-induced colitis is, despite its striking simplicity, such a valuable model of IBD.

Acknowledgements We thank Nadja Sandholzer for excellent animal husbandry. This work is part of the PhD thesis of PD and CM at the University of Munich. This work was supported by the Deutsche Krebshilfe (Max Eder Research Grant to MS), the Deutsche Forschungsgemeinschaft (GK 1202) to MS and SE, GK 1202 student grant to PD, and En 169/7-2 to SE.

Competing interests None.

Provenance and peer review Not commissioned; externally peer reviewed. 


\section{REFERENCES}

1. Podolsky DK. Inflammatory bowel disease. N Engl J Med 2002;347:417-29.

2. Okayasu I, Hatakeyama S, Yamada M, et al. A novel method in the induction of reliable experimental acute and chronic ulcerative colitis in mice. Gastroenterology 1990;98:694-702

3. Dieleman LA, Ridwan BU, Tennyson GS, et al. Dextran sulfate sodium-induced colitis occurs in severe combined immunodeficient mice. Gastroenterology 1994;107: 1643-52.

4. Ishiguro Y. Mucosal proinflammatory cytokine production correlates with endoscopic activity of ulcerative colitis. J Gastroenterol 1999;34:66-74.

5. Monteleone G, Trapasso F, Parrello T, et al. Bioactive IL-18 expression is up-regulated in Crohn's disease. J Immunol 1999;163:143-7.

6. Sartor RB. Cytokines in intestinal inflammation: pathophysiological and clinical considerations. Gastroenterology 1994;106:533-9.

7. Al-Sadi RM, Ma TY. IL-1beta causes an increase in intestinal epithelial tight junction permeability. J Immunol 2007;178:4641-9.

8. Kwon KH, Murakami A, Hayashi R, et al. Interleukin-1beta targets interleukin-6 in progressing dextran sulfate sodium-induced experimental colitis. Biochem Biophys Res Commun 2005;337:647-54.

9. Siegmund B, Lehr HA, Fantuzzi G, et al. IL-1 beta-converting enzyme (caspase-1) in intestinal inflammation. Proc Natl Acad Sci USA 2001;98:13249-54

10. Fritz JH, Ferrero RL, Philpott DJ, et al. Nod-like proteins in immunity, inflammation and disease. Nat Immunol 2006;7:1250-7.

11. Hugot JP, Chamaillard M, Zouali $\mathrm{H}$, et al. Association of NOD2 leucine-rich repeat variants with susceptibility to Crohn's disease. Nature 2001;411:599-603.

12. Ogura $\mathbf{Y}$, Bonen DK, Inohara N, et al. A frameshift mutation in NOD2 associated with susceptibility to Crohn's disease. Nature 2001;411:603-6.

13. Maeda S, Hsu LC, Liu H, et al. Nod2 mutation in Crohn's disease potentiates NF-kappaB activity and IL-1beta processing. Science 2005;307:734-8.

14. van Beelen AJ, Zelinkova Z, Taanman-Kueter EW, et al. Stimulation of the intracellular bacterial sensor NOD2 programs dendritic cells to promote interleukin-17 production in human memory T cells. Immunity 2007;27:660-9.

15. Masters SL, Simon A, Aksentijevich I, et al. Horror autoinflammaticus: the molecular pathophysiology of autoinflammatory disease. Annu Rev Immunol 2009;27:621-68

16. Villani AC, Lemire $M$, Fortin $G$, et al. Common variants in the NLRP3 region contribute to Crohn's disease susceptibility. Nat Genet 2009;41:71-6.

17. Schoultz I, Verma D, Halfvarsson J, et al. Combined polymorphisms in genes encoding the inflammasome components NALP3 and CARD8 confer susceptibility to Crohn's disease in Swedish men. Am J Gastroenterol 2009:104:1180-8.

18. Prescott NJ, Fisher SA, Franke A, et al. A nonsynonymous SNP in ATG16L1 predisposes to ileal Crohn's disease and is independent of CARD15 and IBD5 Gastroenterology 2007;132:1665-71.

19. Rioux JD, Xavier RJ, Taylor KD, et al. Genome-wide association study identifies new susceptibility loci for Crohn disease and implicates autophagy in disease pathogenesis. Nat Genet 2007;39:596-604.

20. Saitoh T, Fujita N, Jang MH, et al. Loss of the autophagy protein Atg16L1 enhances endotoxin-induced IL-1beta production. Nature 2008:456:264-8.
21. Hornung V, Bauernfeind $F$, Halle $A$, et al. Silica crystals and aluminum salts activate the NALP3 inflammasome through phagosomal destabilization. Nat Immunol 2008;9:847-56.

22. Martinon F, Petrilli V, Mayor A, et al. Gout-associated uric acid crystals activate the NALP3 inflammasome. Nature 2006:440:237-41.

23. Bauer C, Loher F, Dauer $M$, et al. The ICE inhibitor pralnacasan prevents DSSinduced colitis in C57BL/6 mice and suppresses IP-10 mRNA but not TNF-alpha mRNA expression. Dig Dis Sci 2007;52:1642-52.

24. Martinon F, Mayor A, Tschopp J. The inflammasomes: guardians of the body. Annu Rev Immunol 2009;27:229-65.

25. Petrilli V, Papin S, Dostert C, et al. Activation of the NALP3 inflammasome is triggered by low intracellular potassium concentration. Cell Death Differ 2007;14 1583-9.

26. Fernandes-Alnemri T, Wu J, Yu JW, et al. The pyroptosome: a supramolecular assembly of ASC dimers mediating inflammatory cell death via caspase-1 activation. Cell Death Differ 2007;14:1590-604.

27. Mariathasan S, Weiss DS, Newton K, et al. Cryopyrin activates the inflammasome in response to toxins and ATP. Nature 2006;440:228-32.

28. Allen IC, Scull MA, Moore CB, et al. The NLRP3 inflammasome mediates in vivo innate immunity to influenza a virus through recognition of viral RNA. Immunity 2009:30:556-65

29. Dostert C, Petrilli V, Van Bruggen $\mathrm{R}$, et al. Innate immune activation through Nalp3 inflammasome sensing of asbestos and silica. Science 2008;320:674-7.

30. Kwon KH, Ohigashi H, Murakami A. Dextran sulfate sodium enhances interleukin-1 beta release via activation of p38 MAPK and ERK1/2 pathways in murine peritoneal macrophages. Life Sci 2007;81:362-71.

31. Siegmund B, Fantuzzi G, Rieder F, et al. Neutralization of interleukin-18 reduces severity in murine colitis and intestinal IFN-gamma and TNF-alpha production. Am J Physiol Regul Integr Comp Physiol 2001:281:R1264-73.

32. McGovern DP, Butler $H$, Ahmad T, et al. TUCAN (CARD8) genetic variants and inflammatory bowel disease. Gastroenterology 2006;131:1190-6.

33. Fisher SA, Mirza MM, Onnie CM, et al. Combined evidence from three large British Association studies rejects TUCAN/CARD8 as an IBD susceptibility gene. Gastroenterology 2007;132:2078-80.

34. Franke A, Rosenstiel P, Balschun $T$, et al. No association between the TUCAN (CARD8) Cys10Stop mutation and inflammatory bowel disease in a large retrospective German and a clinically well-characterized Norwegian sample. Gastroenterology 2007;132:2080-1.

35. Agostini L, Martinon F, Burns K, et al. NALP3 forms an IL-1beta-processing inflammasome with increased activity in Muckle-Wells autoinflammatory disorder. Immunity 2004;20:319-25.

36. Bouchier-Hayes L, Conroy $\mathrm{H}$, Egan $\mathrm{H}$, et al. CARDINAL, a novel caspase recruitment domain protein, is an inhibitor of multiple NF-kappa B activation pathways. J Biol Chem 2001;276:44069-77.

37. Razmara $\mathbf{M}$, Srinivasula SM, Wang L, et al. CARD-8 protein, a new CARD family member that regulates caspase-1 activation and apoptosis. J Biol Chem 2002;277 13952-8.

38. Fukata M, Chen A, Vamadevan AS, et al. Toll-like receptor-4 promotes the development of colitis-associated colorectal tumors. Gastroenterology 2007;133 1869-81. 


\section{GUT Colitis induced in mice with dextran sulfate sodium (DSS) is mediated by the NLRP3 inflammasome}

Christian Bauer, Peter Duewell, Christine Mayer, et al.

Gut 2010 59: 1192-1199 originally published online May 4, 2010

doi: 10.1136/gut.2009.197822

Updated information and services can be found at:

http://gut.bmj.com/content/59/9/1192.full.html

These include:

Data Supplement "Web only appendix"

http://gut.bmj.com/content/suppl/2010/05/25/gut.2009.197822.DC1.html

References This article cites 38 articles, 8 of which can be accessed free at: http://gut.bmj.com/content/59/9/1192.full.html\#ref-list-1

Article cited in:

http://gut.bmj.com/content/59/9/1192.full.html\#related-urls

Email alerting Receive free email alerts when new articles cite this article. Sign up in service the box at the top right corner of the online article.

Notes

To request permissions go to:

http://group.bmj.com/group/rights-licensing/permissions

To order reprints go to:

http://journals.bmj.com/cgi/reprintform

To subscribe to BMJ go to:

http://group.bmj.com/subscribe/ 